IAU Colloquium 164: Radio Emission from Galactic and Extragalactic Compact Sources

ASP Conference Series, Vol. 144, 1998

J. A. Zensus, G. B. Taylor, \& J. M. Wrobel (eds.)

\title{
PKS 0405-385 as Seen by the ISM Telescope
}

L. L. Kedziora-Chudczer \& M. A. Walker

Research Centre for Theoretical Astrophysics, The University of Sydney, NSW, Australia

D. L. Jauncey, M. H. Wieringa, J. E. Reynolds \& A. K. Tzioumis

Australia Telescope National Facility, CSIRO, Sydney NSW, Australia

G. D. Nicolson

Hartebeesthoek Radio Astronomy Observatory, Krugersdorp, South Africa

Abstract. We have found large amplitude intraday variability in the radio quasar PKS 0405-385 on timescales less than an hour. If intrinsic to the source, the inferred brightness temperature is $\mathrm{T}_{\mathrm{B}} \sim 10^{21} \mathrm{~K}$, far exceeding the inverse Compton limit for a static synchrotron source. We argue that our data are in agreement with interstellar scintillations of a source component which is $<5 \mu$ arcsec in size.

\section{Observations}

The ATCA IDV Survey (Kedziora-Chudczer et al., these Proceedings, p. 271) of 125 compact, flat spectrum southern radio sources at four frequencies $(1.4,2.4$, 4.8 , and $8.6 \mathrm{GHz}$ ) led to the discovery of extremely rapid and large amplitude variations in the $z=1.285$ quasar PKS 0405-385 (Kedziora-Chudczer et al. 1996).

The IDV of PKS 0405-385 was first observed in Nov 1993 as a part of the IDV Survey, when strong and rapid changes (17\% peak-to-peak within 24 hours) were seen. Continuous monitoring in June 1996 with the ATCA revealed large amplitude, rapid, quasiperiodic variations. These variations were immediately confirmed by data taken with the $26 \mathrm{~m}$ telescope of the Hartebeesthoek Radio Astronomy Observatory (HARTRAO) at 5.0 and $8.4 \mathrm{GHz}$. Figure 1 shows the data from June 1996 where the excellent agreement in the ATCA and HARTRAO flux density measurements is immediately apparent.

Remarkably, these variations persisted only for several weeks. They were present at full strength on July 2 and entirely absent on July 21 . The flux

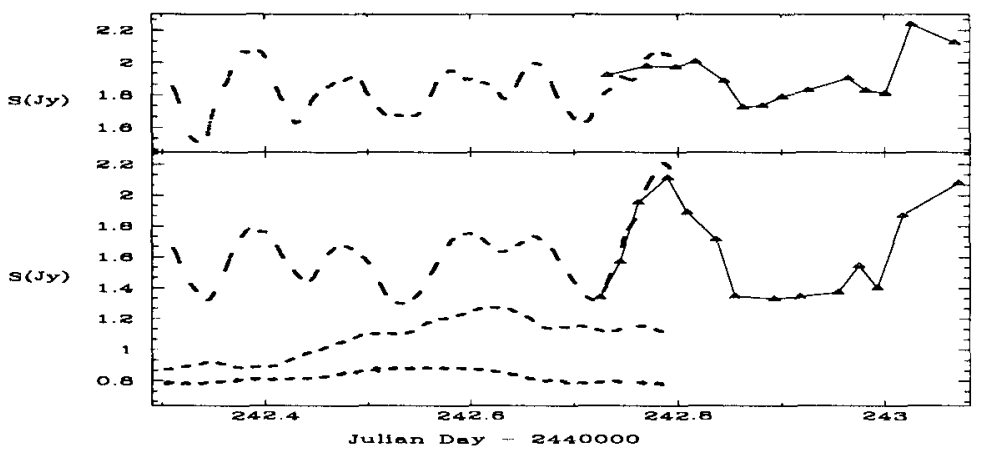

Figure 1. Combined ATCA and HARTRAO light-curve measured on the 1996 June 8 at four frequencies: $8.6,4.8,2.4$, and $1.4 \mathrm{GHz}$, from top to bottom, respectively. ATCA data are plotted as circles, HARTRAO data as triangles. 
density of the source settled on a value which, at each frequency, was close to the maximum values seen during the variable phase. Analysis of sparse ATCA archival data indicates that the rapid variability is episodic on timescales of weeks to months.

\section{Interpretation}

The variability brightness temperature of PKS 0405-385 is $\mathrm{T}_{\mathrm{b}} \sim 10^{21} \mathrm{~K}$. For a synchrotron, relativistically beamed source, this requires a Doppler factor $\delta \sim 10^{3}$. In any model of such rapid flux modulations intrinsic to the source the ultra-relativistic transverse motions are required (see a review by Wagner, these Proceedings, p. 257). We interpret the observed variations in PKS 0405 -385 as due to interstellar scintillations, ISS, in the Galactic interstellar medium (Rickett 1990), where the timescales can be reproduced with smaller transverse velocities, $\sim 50 \mathrm{~km} \mathrm{~s}^{-1}$ (Kedziora-Chudczer et al. 1997). Using the Taylor \& Cordes (1993) model of the ISM, we obtain the good fit shown in Figure 2, to the observed variability spectrum. Within this model the upper limit to the

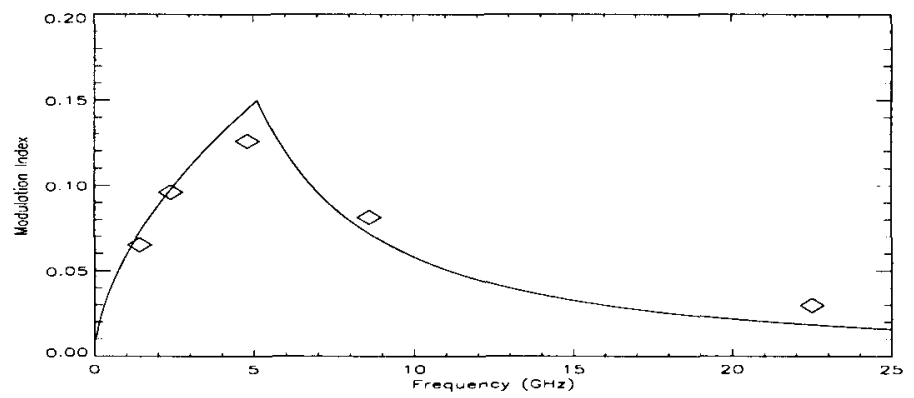

Figure 2. The fit of ISS model (Walker, these Proceedings, p. 285) to the variability spectrum (fractional rms variations vs. frequency) of PKS 0405-385

angular size of the ultra-compact component is 5 microarcsec (the VLBI limit is $<1$ milliarcsec), it has a constant fraction, $15 \%$, of the source flux density at all frequencies, and a brightness temperature of $\sim 10^{14} \mathrm{~K}$. The high $\mathrm{T}_{b}$ indicates strong beaming may be taking place. However, given the episodic nature of the observed variability, it is possible that the inverse Compton limit may be exceeded for a short time (Slysh 1992). We suggest that the variations ceased when the ultra-compact component expanded to become large enough to stop the scintillations.

\section{References}

Kedziora-Chudczer, L. L., et al. 1996. IAU Circ. 6418, 1.

Kedziora-Chudczer, L. L., et al. 1997. ApJ, 490 L9-12.

Rickett, B. J. 1990. ARA\&A, 28, 561-605.

Slysh, V. L. 1992. ApJ, 391, 453-455.

Taylor, J. H., \& Cordes, J. M. 1993. ApJ, 411, 674-677. 